\title{
Management of Latent Tuberculosis Infection: A Key Step in the Direction of TB Elimination from the World
}

\author{
Amit S*, Puneet A and Prabhpreet S \\ Senior Chest Physician, National Institute of Tuberculosis and Respiratory Diseases, India
}

*Corresponding author: Amit Sharma, Senior Chest Physician, National Institute of Tuberculosis and Respiratory Diseases, New Delhi, India, Tel: 9871935758; Email: dramitsharma55@yahoo.co.in

\section{Latent Tuberculosis Infection (LTBI)}

Latent tuberculosis infection (LTBI) is the presence of Mycobacterium tuberculosis in the body without signs and symptoms, or radiographic or bacteriologic evidence of tuberculosis (TB) disease. Approximately one-third of the world's population is infected [1]. Developed countries have been successful in lowering down TB incidence by targeted testing of high risk population, whereas poor countries, whose main objective is to cut down TB transmission by primarily treating active cases, may have as much as $50 \%$ of the population infected, making LTBI treatment practically impossible. Yet targeted testing of high risk populations may prove useful as exemplified by the successful treatment of LTBI in HIV infected individuals $[2,3]$.

\section{Diagnosis of Latent TB Infection}

The lack of diagnostic tests for LTBI further compounds the problem of its successful treatment. The Tuberculin Skin Test (TST), used since a long time for the diagnosis of LTBI, is neither adequately specific nor sensitive, making the decision to administer prolonged costly treatment on its basis alone, highly impractical. Newer diagnostic tests such as Interferon Gamma Release Assay (IGRA) require a single visit and are unaffected by BCG vaccination and most Non-Tubercular Mycobacterial (NTM) infections. However, TST is still preferred in children less than five years and in developing countries with limited resources [4,5]. It is of vital importance to rule out active TB before starting LTBI treatment in patients with a positive IGRA/TST, who are at greater risk for developing active disease. This includes patients receiving corticosteroids, immunosuppressants, HIV-infected and juvenile contacts of sputum-positive index cases with a documented recent TST conversion $[4,6]$.

\section{Treatment of LTBI}

Even as the diagnosis of LTBI is no easy task, its treatment is no less problematic. Use of potentially toxic drugs for extended intervals of time poses difficulty both to patients (mostly asymptomatic) and providers. Studies have demonstrated that Isoniazid (INH) taken for at least 6 months in persons with LTBI reduced subsequent TB incidence by 25 to 92 per cent, the differences in effectiveness largely explained by differences in treatment completion [7]. Use of INH, however, in LTBI treatment is fraught with difficulties. Its long duration of administration (6 to 12 months) coupled with potentially lethal albeit uncommon adverse effects such as hepatitis reduce its acceptability both to patients and providers alike $[8,9]$.

\section{Different Regimens for Treatment of LTBI}

The International Union Against Tuberculosis (IUAT) trial, conducted in Eastern Europe, showed that participants, who completed 6 and 12 months of INH, had 69 and 93 per cent reduction in active TB, respectively [10]. However, completion of the 12 month regimen was much less than the 6 month regimen, thus prompting the American Thoracic Society (ATS) in 2000 to recommend 9 months INH with estimated efficacy of 90 per cent as the acceptable regimen [4]. Adverse effects, especially hepatitis, may be difficult to detect and can lead to fatality which may be as high as 1 per cent in older patients [11].

The problems with INH have stimulated development and evaluation of several shorter regimens. Several randomized trials conducted to compare 6 to 12 months INH with 2 months of Rifampicin (RIF) and Pyrazinamide (PZA) in HIV infected patients demonstrated equivalent efficacy [12]. 


\section{Open Access Journal of Pulmonary \& Respiratory Sciences}

In 2000, ATS recommended use of 2 months RIF-PZA with a strong recommendation for use in HIV infected persons and a conditional recommendation for non-HIV infected persons [4]. This led to widespread use of 2RIF-PZA,but was quickly followed by reports of serious hepatotoxicity and death, leading to revision of recommendations with cautious use in HIV infected $[13,14]$.

\section{Newer Regimens Including Regimes for Drug Resistant TB}

One of the promising new drugs being tested for the treatment of LTBI is Rifapentine (RPT), a cyclopentylsubstituted rifamycin that is as effective as rifampicin, but whose serum half-life is five times that of rifampicin, thus permitting weekly dosing. The INH-RPT combined regime was investigated and the first results have been published [15]. These results show that once-weekly, three month regime of INH-RPT (900 $\mathrm{mg}$ each) was as effective as 9 months of isoniazid alone in preventing tuberculosis and had a higher treatment-completion rate. Results of further trials with RPT for treatment of LTBI are awaited.

Rifabutin may be substituted for RIF in HIV-positive patients at risk for INH-resistant TB owing to its lower interaction with anti-retroviral drugs as compared to RIF. The 6-monthly regime of PZA and a quinolone, recommended for LTBI treatment of MDR-TB contacts, has been shown to have very poor completion rates due to high toxicity, thus prompting the use of monotherapy with Levofloxacin or Moxifloxacin, the latter showing special promise on account of published literature showing its equivalence to $\mathrm{INH}$ $[16,17]$.

In their detailed meta-analysis of 10 randomised controlled trials consisting of 10,717 HIV-negative adults and children, S K Sharma et al concluded that shortened prophylactic regimens using rifampicin alone did not demonstrate higher rates of active TB when compared to longer regimens with INH. A weekly regimen of rifapentine plus INH had higher completion rates with less liver toxicity but more treatment discontinuation due to adverse events than with INH [18]. Recently WHO has published detailed guidelines for treatment of LTBI regardless of HIV status, which gives the options for 6-month or 9-months INH, 3-month RPT plus INH once weekly or 3-month of Rif-INH daily [19].

\section{LTBI Treatment in Context of India}

India, with one-fifth of the global burden of TB, has 40 per cent of the population infected, with 1.5 per cent annual risk of developing latent infection $[20,21]$. The priority is to treat sputum-positive patients in order to interrupt the transmission in TB. Treating 40 per cent of the population for LTBI based on TST or IGRA is neither rational nor practicable, thus emphasizing the need for a focussed approach. The most obvious group for LTBI treatment would include high-risk patients such as those receiving corticosteroids, immunosuppressants, HIV-infected and juvenile contacts of sputum-positive index cases, showing recent TST conversion.

Another major concern in LTBI treatment is development of drug resistance, the main reason being improper administration and/or dosing, which can be prevented by strict monitoring, good education and rigorous follow-up. A second reason for the development of resistance could be due to partial treatment of active TB masquerading as LTBI, which can be avoided by a thorough clinical assessment, based on sound history and appropriate investigations such as chest radiograph and sputum testing, before starting LTBI treatment.

Finally, it is the responsibility of the health care provider team to ensure that patient complies with treatment once the decision to treat LTBI with a suitable regime on an individual basis has been taken.

\section{References}

1. WHO (2010) Global tuberculosis control, Surveillance, planning, financing.

2. Hawken MP, Meme HK, Elliott LC, Chakaya JM, Morris JS, et al. (1997) Isoniazid preventive therapy for tuberculosis in HIV-1-infected adults: results of a randomized controlled trial. AIDS 11(7): 875-882.

3. Akolo C, Ifedayo A, Shepperd S, Volmink J (2010) Treatment of latent tuberculosis infection in HIV infected persons. Cochrane Database Syst Rev 2010(1).

4. Targeted tuberculin testing and treatment of latent tuberculosis infection. This official statement of the American Thoracic Society was adopted by the ATS Board of Directors, July 1999. This is a Joint Statement of the American Thoracic Society (ATS) and the Centers for Disease Control and Prevention (CDC). This statement was endorsed by the Council of the Infectious Diseases Society of America. (IDSA), September 1999, and the sections of this statement. Am J Respir Crit Care Med 161(4 Pt 2): S221-S247.

5. Mazurek GH, Jereb J, Vernon A, LoBue P, Goldberg S, et al. (2010) Updated guidelines for using Interferon Gamma Release Assays to detect Mycobacterium tuberculosis infection-United States, 2010. MMWR Recomm Rep 59(RR-5): 1-25. 


\section{Open Access Journal of Pulmonary \& Respiratory Sciences}

6. Horsburgh CR (2004) Priorities for the treatment of latent tuberculosis infection in the United States. $\mathrm{N}$ Engl J Med 350(20): 2060-2067.

7. Ferebee SH (1970) Controlled chemoprophylaxis trials in tuberculosis. A general review. Bibl Tuberc 26: 28106.

8. Dash LA, Comstock GW, Flynn JP (1980) Isoniazid preventive therapy: Retrospect and prospect. Am Rev Respir Dis 121(6): 1039-1044.

9. Miller B, Snider DE (1987) Physician noncompliance with tuberculosis preventive measures. Am Rev Respir Dis 135(1): 1-2.

10. International Union against Tuberculosis Committee on Prophylaxis (1982) Efficacy of various durations of isoniazid preventive therapy for tuberculosis: five years of follow-up in the IUAT trial. International Union against Tuberculosis Committee on Prophylaxis. Bull World Health Organ 60(4): 555-564.

11. Kopanoff DE, Snider DE, Caras GJ (1978) Isoniazidrelated hepatitis: a U.S. Public Health Service cooperative surveillance study. Am Rev Respir Dis 117(6): 991-1001.

12. Gao XF, Wang L, Liu GJ, Wen J, Sun X, et al. (2006) Rifampicin plus pyrazinamide versus isoniazid for treating latent tuberculosis infection: a meta-analysis. Int J Tuberc Lung Dis 10(10): 1080-1090.

13. Centers for Disease Control and Prevention (CDC) (2001) Fatal and severe hepatitis associated with rifampin and pyrazinamide for the treatment of latent tuberculosis infection--New York and Georgia, 2000. MMWR Morb Mortal Wkly Rep 50(15): 289-291.

14. Centers for Disease Control and Prevention (CDC)
(2001) Update: Fatal and severe liver injuries associated with rifampin and pyrazinamide for latent tuberculosis infection, and revisions in American Thoracic Society/ CDC recommendations--United States, 2001. MMWR Morb Mortal Wkly Rep 50(34): 733-735.

15. Sterling TR, Villarino ME, Borisov AS, Shang N, Gordin F, et al. (2011) Three months of rifapentine and isoniazid for latent tuberculosis infection. N Engl J Med 365(23): 2155-2166.

16. Younossian $\mathrm{AB}$, Rochat $\mathrm{T}$, Ketterer JP, Wacker J, Janssens JP, et al. (2005) High hepatotoxicity of pyrazinamide and ethambutol for treatment of latent tuberculosis. Eur Respir J 26(3): 462-464.

17. Dorman SE, Johnson JL, Goldberg S, Muzanye G, Padayatchi N, et al. (2009) Substitution of moxifloxacin for isoniazid during intensive phase treatment of pulmonary tuberculosis. Am J Respir Crit Care Med 180(3): 273-280.

18. Sharma SK, Sharma A, Kadhiravan T, Tharyan P (2013) Rifamycins (rifampicin, rifabutin and rifapentine) compared to isoniazid for preventing tuberculosis in HIV-negative people at risk of active TB. Cochrane Database Syst Rev 2013(7): CD007545.

19. WHO consolidated Guidelines on tuberculosis: module 1: prevention: tuberculosis preventive treatment.

20. Chadha VK, Kumar P, Jagannatha PS, Vaidyanathan PS, Unnikrishnan KP (2005) Average annual risk of tuberculous infection in India. Int J Tuberc Lung Dis 9(1): 116-118.

21. Khatri GR, Frieden TR (2002) Controlling tuberculosis in India. N Engl J Med 347(18): 1420-1425. 